\title{
Speckle correlation functions applied to surface plasmons
}

\author{
Frerik van Beijnum, ${ }^{1}$ Jeroen Sirre ${ }^{1}$ Chris Rétif, ${ }^{2}$ and Martin P. van Exter ${ }^{1}$ \\ ${ }^{1}$ Huygens Laboratory, Leiden University, P.O. Box 9504, NL-2300 RA Leiden, The Netherlands \\ ${ }^{2}$ FOM Institute for Atomic and Molecular Physics, Science Park 104, NL-1098 XG Amsterdam, The Netherlands
}

(Received 19 October 2011; published 23 January 2012)

\begin{abstract}
The optical intensity transmitted through a random pattern of subwavelength holes in a metal film exhibits a speckle pattern. We study the variation of this speckle pattern as a function of wavelength. We find that the resulting speckle correlation function (SCF) separates into a wavelength-dependent part and a wavelength-independent background. The wavelength dependence is caused by surface plasmons excited at one hole and coupled out at another hole, while the constant background originates from light transmitted directly through the holes. By analyzing the SCF for a set of samples of varying hole density, we find the propagation length of the surface plasmons and the scattering losses induced by the holes.
\end{abstract}

DOI: 10.1103/PhysRevB.85.035437

PACS number(s): 73.20.Mf, 42.25.Dd, 78.67.-n, 42.30.Ms

\section{INTRODUCTION}

Structuring materials on a scale comparable to or smaller than the wavelength of light allows control over their optical properties in an unprecedented way. Famous examples are photonic crystals, metal hole arrays, and metamaterials. As the complexity of these structures increases, it becomes more challenging to understand their physics. For example, to quantitatively model the extraordinary optical transmission (EOT) ${ }^{1}$ of metal hole arrays a large set of parameters that need to be calculated numerically is required. ${ }^{2}$ Also the transmission of light through a single hole in a metal film is surprisingly complex. ${ }^{3}$

In contrast, disordered media can be characterized relatively easy. By studying the variations of speckle as a function of wavelength or angle, speckle correlation functions (SCFs) can be calculated analytically. ${ }^{4}$ These functions, which can also be measured, ${ }^{5-9}$ provide insight in the dwell time inside a medium. Only three quantities are needed to describe the SCF in volume scattering: the transport mean free path, the energy velocity, ${ }^{10}$ and the sample thickness.

In this work we apply the framework of speckle correlation functions to surface plasmons. To this extent, we study the optical transmission through random patterns of subwavelength holes. These random patterns were previously studied using a broadband source, ${ }^{11,12}$ but when illuminated with laser light, the transmitted intensity exhibits a speckle pattern. ${ }^{13}$ The intensity in this speckle pattern has two contributions: light that is directly transmitted through the holes and light transmitted via surface waves. By studying the change of these speckle patterns as we scan the wavelength of the laser we can record a SCF. Because of the two different contributions to the speckle intensity, we expect that these correlation functions are different from the ones found in three-dimensional random media.

From the SCF we hope to infer a propagation length. As it is unclear whether the losses are dominated by absorption or scattering, we study a set of samples with different hole densities. We analyzed a set of nine samples for which the hole density is $1 /\left(q a_{0}^{2}\right)$, where $a_{0}=450 \mathrm{~nm}$ and $q=$ $1,2,3,4,9,16,25,36,81$. Each sample covers a square area of $400 \mu \mathrm{m} \times 400 \mu \mathrm{m}$. The average side length of these square holes is $125 \pm 5 \mathrm{~nm}$. The layer structure is as follows: on the glass substrate we subsequently deposited $150 \mathrm{~nm}$ of gold and $20 \mathrm{~nm}$ of chrome, and we then perforated this metal layer. The function of the chrome layer is to damp the surface plasmons on the gold-air interface, limiting the analysis to one interface. Figure 1 shows scanning electron microscopy (SEM) images of three of the studied samples, with densities of $0.19,1.2$, and $4.9 \mu \mathrm{m}^{-2}$, which is, $q=25, q=4$, and $q=1$, respectively.

\section{THEORY}

Before showing the experimental results, we will first derive an expression for the expected correlation function. In the far field the speckle intensity is a function of wavelength and angle: $I(\lambda, \vec{\theta})$. We define the speckle correlation function as follows:

$$
C \equiv \frac{\left\langle I\left(\lambda_{0}, \vec{\theta}_{0}\right) I\left(\lambda_{1}, \vec{\theta}_{1}\right)\right\rangle}{\left\langle I\left(\lambda_{0}, \vec{\theta}_{0}\right)\right\rangle\left\langle I\left(\lambda_{1}, \vec{\theta}_{1}\right)\right\rangle}-1,
$$

where $\langle\cdot\rangle$ denotes ensemble averaging. ${ }^{4}$ We can rewrite the intensity correlation function in terms of the optical fields $E(\lambda, \vec{\theta})$ using Isserlis's theorem ${ }^{14}$ for Gaussian random variables: $^{15}$

$$
C=\frac{\left|\left\langle E\left(\lambda_{0}, \vec{\theta}_{0}\right) E^{*}\left(\lambda_{1}, \vec{\theta}_{1}\right)\right\rangle\right|^{2}}{\left\langle I\left(\lambda_{0}, \vec{\theta}_{0}\right)\right\rangle\left\langle I\left(\lambda_{1}, \vec{\theta}_{1}\right)\right\rangle},
$$

where the asterisk $\left(^{*}\right)$ is the complex conjugate

When measuring in the far field, at an angle $\vec{\theta}$, the light transmitted through the randomly positioned holes is a sum over all illuminated holes $E_{i}$. Depending on the position $\vec{x}_{i}$,
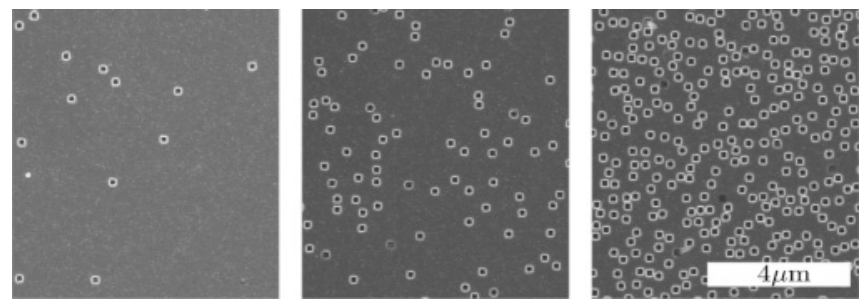

FIG. 1. SEM images of three of the nine studied samples. From left to right the hole density is $0.19,1.2$, and $4.9 \mu \mathrm{m}^{-2}$. 
the field from each hole acquires a random phase: $\vec{k}_{\|}(\lambda) \vec{x}_{i}$, with $\left|\vec{k}_{||}(\lambda)\right|=2 \pi \sin (|\vec{\theta}|) / \lambda$. Hence the correlation is

$$
C \propto\left|\sum_{i, i^{\prime}}\left\langle E_{i}\left(\lambda_{0}\right) E_{i^{\prime}}^{*}\left(\lambda_{1}\right) e^{i\left[\vec{k}_{\|}\left(\lambda_{0}\right) \vec{x}_{i}-\vec{k}_{\|}\left(\lambda_{1}\right) \vec{x}_{i^{\prime}}\right]}\right\rangle\right|^{2},
$$

where the proportionality sign indicates that normalization is now omitted. Calculations can be performed with this expression, but the decorrelation from $e^{i\left[\vec{k}_{\|}\left(\lambda_{0}\right) \vec{x}_{i}-\vec{k}_{\|}\left(\lambda_{1}\right) \vec{x}_{i^{\prime}}\right]}$ may have a strong influence on the correlation function. ${ }^{16}$ Hence, we take $\vec{k}_{\|}\left(\lambda_{0}\right)=\vec{k}_{\|}\left(\lambda_{1}\right)$, such that the exponent becomes 1 for $\vec{x}_{i}=\vec{x}_{i^{\prime}}$. The phase of this term is random when $\vec{x}_{i} \neq \vec{x}_{i^{\prime}}$ and $\vec{k}_{\|}$is sufficiently large, i.e., if we are outside the zeroth diffraction order. ${ }^{13}$ Hence, after ensemble averaging, the terms with $i \neq i^{\prime}$ are zero, and therefore the double sum over $i$ and $i^{\prime}$ reduces to a single sum.

The field $E_{i}$ is a sum of a direct contribution $E_{d, i}$ and a contribution via surface waves $E_{s, i}$. The field $E_{s, i}$ consists of contributions from all neighboring holes. Hence the amplitude and phase are random and depend on the position of the surrounding holes. Although the phase is random, it changes gradually as the excitation wavelength changes. We can now evaluate Eq. (3) further by inserting $E_{i}(\lambda)=E_{d, i}+E_{s, i}(\lambda)$ and taking the ensemble average:

$$
C=\frac{\left|\left\langle\left|E_{d}\right|^{2}\right\rangle+\left\langle E_{s}\left(\lambda_{0}\right) E_{s}^{*}\left(\lambda_{1}\right)\right\rangle\right|^{2}}{\left\langle I_{\mathrm{tot}}\right\rangle^{2}},
$$

with $\left\langle I_{\text {tot }}\right\rangle=\left\langle I_{d}\right\rangle+\left\langle I_{s}\right\rangle$, where $\left\langle I_{d}\right\rangle$ and $\left\langle I_{s}\right\rangle$ are assumed to be wavelength independent for the clarity of the expressions. Please note that the summation and the $i$ dependence disappear because the summation is replaced by the number of holes times the ensemble average. Also the ensemble average of the cross terms is zero, $\left\langle E_{d, i} E_{s, i}^{*}+E_{d, i}^{*} E_{s, i}\right\rangle=0$, because $E_{s, i}$ and $E_{d, i}$ are independent and the ensemble average of $E_{s, i}$ is zero. Equation (4) is an essential result: the direct transmission is observable as a constant background correlation, while the contribution from surface waves does decorrelate in a limited wavelength range.

Next we calculate the wavelength-dependent correlation caused by the surface waves. For simplicity we neglect contributions from the quasicylindrical wave ${ }^{17}$ and Norton wave $^{2,18,19}$ and surface plasmon polaritons (SPP), which we will simply denote as SPP or surface plasmons. We consider a particular hole $i$ and write the total SPP field as a sum of contributions from all the surrounding holes $j$. We describe the SPP propagation as a two-dimensional surface wave. As it propagates, it can be absorbed or scattered out by a hole such that

$$
E_{s, i}=\sum_{j} \frac{A_{0}\left(\phi_{j}\right)}{\sqrt{r_{i j}}} e^{\left(-\sigma \rho-\operatorname{Im} k_{\mathrm{spp}}+i \operatorname{Re} k_{\mathrm{spp}}\right) r_{i j}},
$$

where $A_{0}(\phi)$ is a prefactor describing the excitation and outcoupling of the field, which has units of $V / \sqrt{m}$ and depends on the angle $\phi$ between the incident polarization and the propagation direction, ${ }^{13,20} r_{i j}$ is the distance between hole $i$ and hole $j, \operatorname{Im} k_{\text {spp }}$ characterizes the loss due to absorption, $\sigma \rho$ characterizes the loss due to scattering, $\sigma$ is the scattering cross section, and $\rho$ is the hole density. We are dealing with a two-dimensional problem, and therefore the scattering cross section is a length instead of an area.

When evaluating $\left\langle E_{s}\left(\lambda_{0}\right) E_{s}^{*}\left(\lambda_{1}\right)\right\rangle$, a double sum is found, for which the cross terms associated with interference originating from different holes $j \neq j^{\prime}$ again average out. We rewrite the phase difference of the remaining terms as $r_{i j} / \lambda_{0}-$ $r_{i j} / \lambda_{1}=r_{i j} \Delta \lambda /\left(\lambda_{0} \lambda_{1}\right)$, as in Ref. 15. We then form concentric rings around hole $i$ to find that the number of holes in each ring scales with the radius $r$. On the other hand, the contribution from these holes scales as $1 / r$ multiplied by an exponential; see Eq. (5). By replacing the sum over neighboring holes for an integral we find

$$
C=\frac{1}{\left\langle I_{\mathrm{tot}}\right\rangle^{2}}\left|\left\langle I_{d}\right\rangle+\frac{\left\langle I_{s}\right\rangle \tilde{\lambda}}{i \Delta \lambda+\widetilde{\lambda}}\right|^{2} .
$$

The width of the Lorentzian,

$$
\tilde{\lambda}=\left(\sigma \rho+\operatorname{Im} k_{\mathrm{spp}}\right) \frac{2 \lambda_{0}}{\operatorname{Re} k_{\mathrm{spp}}},
$$

combines the scattering losses $(\propto \sigma \rho)$ and the ohmic losses $(\propto$ $\left.\operatorname{Im} k_{\mathrm{spp}}\right)$. The relative strength of the Lorentzian is determined by the ratio $\left\langle I_{s}\right\rangle /\left\langle I_{d}\right\rangle$, where $\left\langle I_{s}\right\rangle$ is given by

$$
\left\langle I_{s}\right\rangle \propto \frac{\rho\left\langle\left|A_{0}(\phi)\right|^{2}\right\rangle_{\phi}}{\sigma \rho+\operatorname{Im} k_{\mathrm{spp}}}
$$

where the proportionality sign indicates that we omitted constant prefactors.

To summarize, we expect that we can measure a correlation function with a background that is independent of wavelength. In addition to this background correlation, a wavelengthdependent part is expected, caused by SPPs propagating on the metal surface. The width of this wavelength-dependent part is proportional to the losses that the SPP experiences. The hole density is a crucial parameter for the correlation function, it determines both the weight of the SPP part and the total loss that the SPP experiences.

\section{EXPERIMENTS}

The experimental setup is as follows: the light of a wavelength tunable Coherent 899 Ti:sapphire laser is led through a single-mode fiber and collimated to a beam with a diameter of a few millimeters. This beam is first polarized and then illuminates a $200-\mu \mathrm{m}$ pinhole that is imaged onto the sample with a magnification of $3 / 8$, leaving a $75-\mu \mathrm{m}$ spot on the sample. This illuminated pinhole ensures that the size and position of the spot on the sample are wavelength independent. The light transmitted through the sample was collected using an aspheric lens $(f=8 \mathrm{~mm})$. The Fourier plane of this lens is imaged with a lens onto an intermediate plane, and this intermediate plane is subsequently imaged onto a CCD. In the intermediate plane we blocked the zeroth-order transmission with a black metal rod, and we selected the polarization parallel to the incident polarization. In all our experiments we scan the laser wavelength from 740 to $810 \mathrm{~nm}$.

We now first study the speckles that we see on the CCD. To derive Eq. (4), it was crucial to keep $\vec{k}_{\|}$constant with wavelength. Experimentally, this is achieved by rescaling the recorded images. In Fig. 2 we show the original and rescaled 


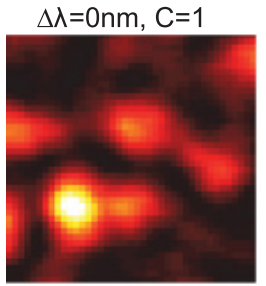

$\Delta \lambda=0 \mathrm{~nm}, \mathrm{C}=1$
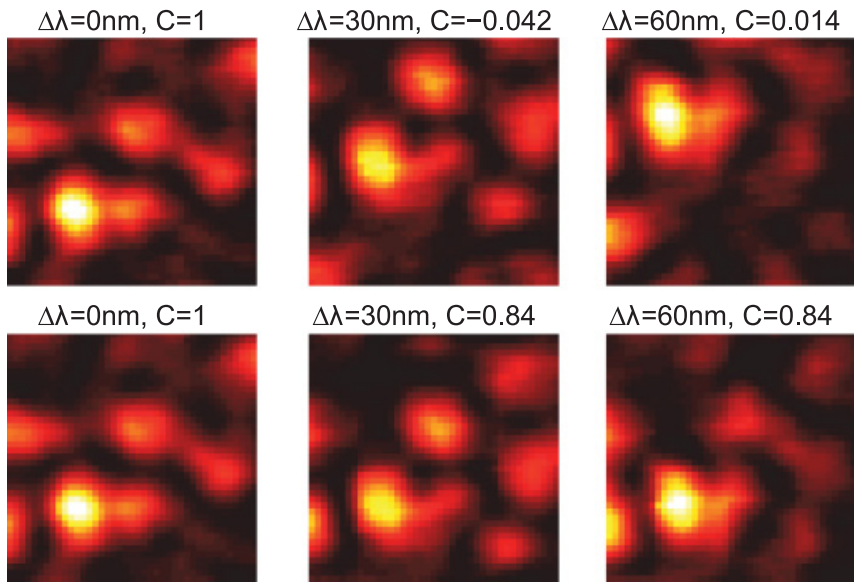

FIG. 2. (Color online) (top) Uncorrected and (bottom) corrected speckles as a function of wavelength detuning. The uncorrected speckle changes shape and gradually shifts upward. After correction the speckle remains approximately in place and only changes shape. The amount of correlation is indicated by $C$.

images as a function of wavelength for a sample of hole density $1.2 \mu \mathrm{m}^{-2}$. The top three images are shown as they are originally recorded. The zeroth-order peak is located below these speckles. As the wavelength is tuned, the speckles move up and change.

In the bottom row in Fig. 2 the three images are corrected such that $\vec{k}_{\| \mid}(\lambda)$ is constant. For these images the speckle shape changes gradually, but the speckles remain at approximately the same position. The effect on the correction is illustrated by the amount of correlation between a reference and the shown images. For the original images the correlation decreases rapidly from 1 to -0.042 and 0.014 ; for the rescaled images the correlation is $1,0.84$, and 0.84 .

For rescaling the images we need to choose a point in the image for which $\vec{k}_{\|}(\lambda)=0$. Although we expected this point to be the zeroth order, we noticed that the correlation between two images recorded at different wavelengths is a few percent larger when we choose this point outside the zeroth order. The origin of this effect has not been resolved so far. For our data processing we used the optimum point outside the zero order.

Now that we know how to rescale the images, we can measure the speckle correlation functions. Figure 3 shows three typical correlation functions for the samples with densities of $4.9,1.2$, and $0.19 \mu \mathrm{m}^{-2}$. The correlation function changes drastically with the hole density. Nonetheless, all measured correlation functions exhibit the predicted behavior: a wavelength-dependent contribution and a constant background correlation. With increasing hole density, the background level decreases while the width of the wavelength-dependent part increases. The decreasing background level is due to the increasing SPP contribution, as our model suggests. The increasing width implies shorter propagation lengths. This may be expected, as the scattering losses are proportional to the hole density.

To quantify our findings we have fitted the measured correlation functions to Eq. (6). Figure 3 shows that the theory describes the data well. The observation that our simple model describes the data might come as a surprise, as we omitted

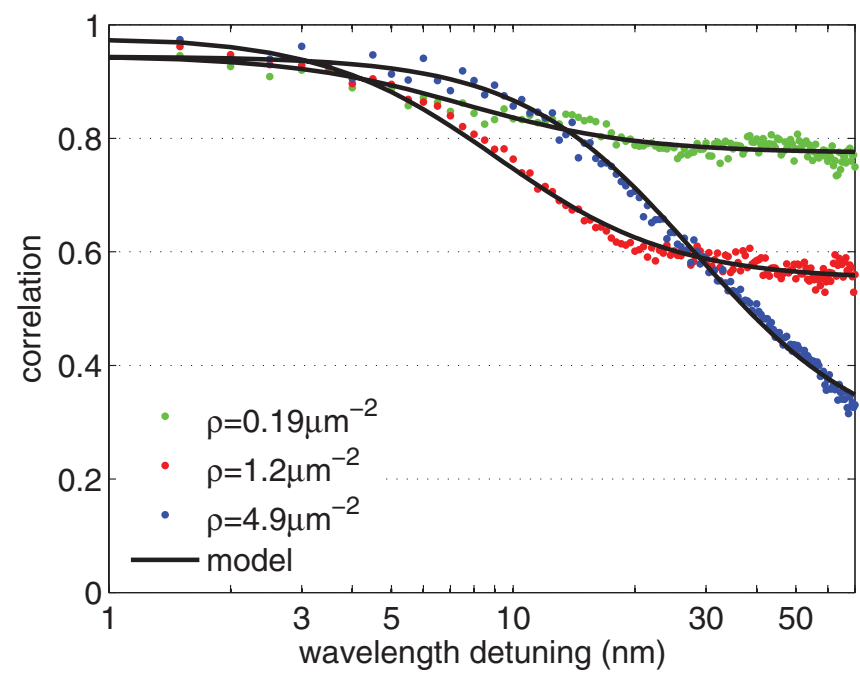

FIG. 3. (Color online) Measured correlation functions for three different hole densities. Each fit function combines a Lorentzian part with a wavelength-independent background. With increasing hole density the Lorentzian becomes wider, while the background decreases.

the wavelength dependence of both $\left\langle I_{d}\right\rangle$ and $\left\langle I_{s}\right\rangle$. Especially for $\left\langle I_{d}\right\rangle$ it is well known that it has a strong wavelength dependence; see, e.g., Ref. 3 for a recent review. There are two ways to interpret our observation of a constant background correlation. First, because we normalize the recorded speckle patterns [see Eq. (1)], a constant background correlation will also be found if the ratio $\left\langle I_{s}\right\rangle /\left\langle I_{d}\right\rangle$ is constant. So far, there is not much literature reported on the wavelength dependence of $\left\langle I_{s}\right\rangle$, but work on hole chains suggests that this is possible. ${ }^{17}$ Second, the normalization makes the effect of the wavelength dependence smaller, as not only the nominator of the correlation changes but also the denominator. For a typical example, with $\left\langle I_{s}\right\rangle /\left\langle I_{d}\right\rangle=1 / 4$ and a reduction of $\left\langle I_{d}\right\rangle$ to $70 \%$ of its original value, the background decreases to $62 \%$ instead of $67 \%$ for the constant background. To see this small decay, the scan range would have to be much larger. Finally, we also have an experimental argument for why the wavelength dependence of $\left\langle I_{d}\right\rangle$ and $\left\langle I_{d}\right\rangle$ are not relevant. If we scan in the opposite direction, namely, 810 to $740 \mathrm{~nm}$, instead of increasing the wavelength, we find practically the same correlation $C(\Delta \lambda)$.

\section{EXTRACTED FIT PARAMETERS}

Two fit parameters are extracted from the fit, the losses $\left(\sigma \rho+\operatorname{Im} k_{\mathrm{spp}}\right)$ and the ratio $\left\langle I_{s}\right\rangle /\left\langle I_{d}\right\rangle$. As seen in Fig. 4(a), the loss increases linearly with hole density, as expected from Eq. (7). However, the loss of the highest-hole-density sample is larger than expected from a linear dependence. For this data point the error margin is also larger than the other data points because the background level could not be fitted properly as the correlation still decreases within our scan range.

We fitted these loss values with a linear function, from which we find an offset of $0.049 \pm 0.002 \mu \mathrm{m}^{-1}$ and the slope $\sigma=26 \pm 2 \mathrm{~nm}$. The cross section is reasonable, considering 

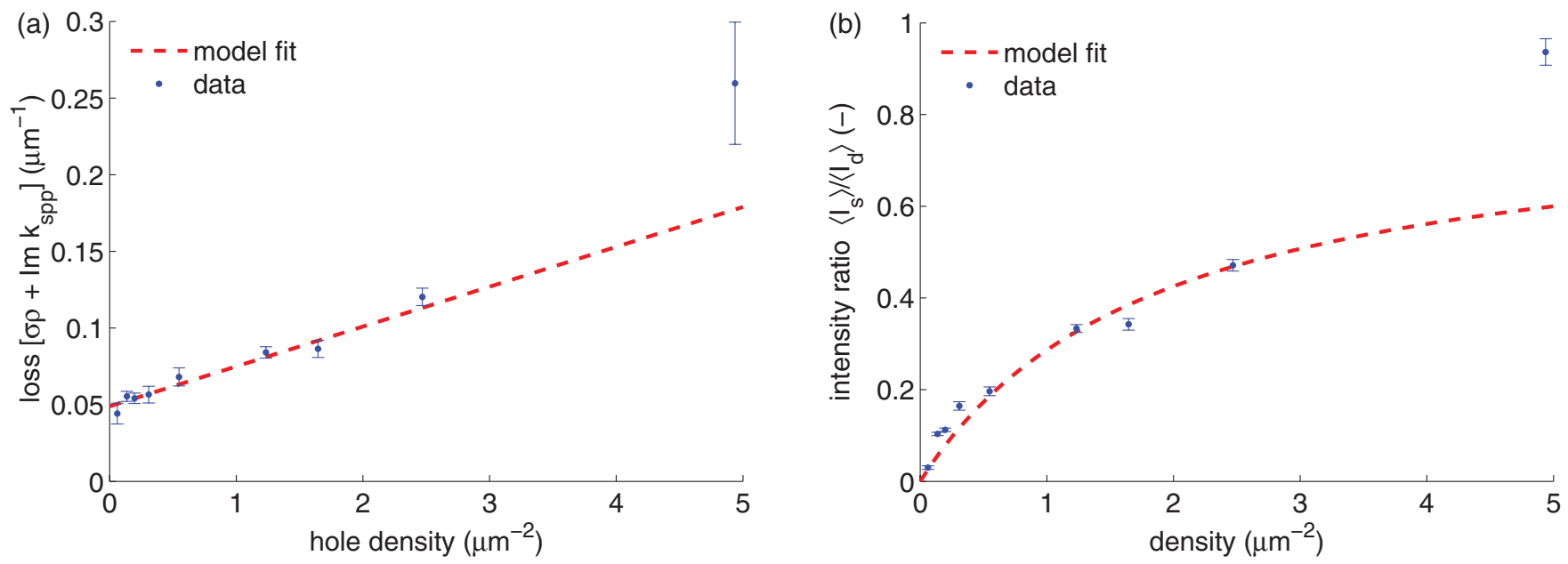

FIG. 4. (Color online) The loss and intensity ratio plotted as a function of hole density. (a) For all samples except the most dense, the loss increases linear with hole density. The loss at zero hole density is the surface-plasmon absorption loss, which corresponds roughly to the literature value. (b) The surface-plasmon contribution $\left\langle I_{s}\right\rangle$ relative to the direct contribution $\left\langle I_{d}\right\rangle$ increases nonlinearly with hole density. Using only the vertical scale as a free parameter, the data can be reasonably fitted to theory.

the hole side length of $125 \pm 5 \mathrm{~nm}$. The offset corresponds reasonably to the values that can be calculated using the dielectric constant of gold at $740 \mathrm{~nm}$ reported by Johnson and Christy ${ }^{21}\left(0.056 \mu \mathrm{m}^{-1}\right)$ and Palik ${ }^{22}\left(0.065 \mu \mathrm{m}^{-1}\right)$.

In Fig. 4(b) the ratio $\left\langle I_{s}\right\rangle /\left\langle I_{d}\right\rangle$ is plotted versus hole density. We observe a nonlinear increase. Equation (8) shows that when $\sigma \rho$ is comparable to $\operatorname{Im} k_{\mathrm{spp}}$, this nonlinear increase is expected. We fitted Eq. (8) to these data, using the values of $\operatorname{Im} k_{\text {spp }}$ and $\sigma$ we just obtained, leaving only the vertical scale as a free parameter. The fit result is reasonable. Interestingly, both the intensities at low hole density and at the largest density are underestimated by the fit.

Nevertheless, from fitting the intensity ratio we conclude that the scattering cross section found in Fig. 4(a) fits the data reasonably well. The high intensity for the most dense sample can imply an extra contribution to the field, which may be caused by the quasicylindrical wave. ${ }^{2,17,19}$ However, to be able to make a sensible judgment about this, the correlation function should be studied theoretically, including and excluding the quasicylindrical wave. Moreover, it would be of great value to use a scanning laser with a larger range to measure the full correlation function for this sample.

Another interesting question that arises from these data is whether the underestimation of the low hole density is related to the Norton wave..$^{2,18,19}$ At the distance probed with these low densities, roughly five propagation lengths $\left(\rho \sigma=\frac{1}{5} \operatorname{Im} k_{\mathrm{spp}}\right)$, the Norton wave should have the same amplitude as the SPP. However, at these distances the field is only $7 \times 10^{-3}$ of its original strength, and we wonder whether these small fields contribute to the decorrelation seen. On the other hand, the Norton wave becomes more than three orders of magnitude larger than the SPP field for a distance of roughly ten propagation lengths. ${ }^{19}$

Besides this set of random patterns with square holes, we have also analyzed a set of patterns of circular holes, with a diameter of $120 \pm 6 \mathrm{~nm}$. For these samples the signal is smaller than for square holes, and hence we could only measure the five most dense samples. The measured correlation functions show a larger background correlation when comparing patterns with the same hole density. From a similar analysis as for the square holes we found a cross section of $18 \pm 1 \mathrm{~nm}$ and an absorption loss of $0.035 \pm 0.002 \mu \mathrm{m}^{-1}$. The loss of the most dense sample is now not as high as found for the square holes, and all measured loss values fit the SPP model.

\section{CONCLUSION AND DISCUSSION}

In conclusion, we have derived a simple expression for the speckle correlation function for random patterns of subwavelength holes. The expression has two contributions, a constant background resulting from the direct transmission of the holes and wavelength-dependent part due to surface plasmons propagating on the surface. The predicted behavior of a constant background and wavelength-dependent part is also seen in the measured correlation function. By fitting the experimental results, we find that the propagation length of the surface plasmon decreases with increasing hole density, as the surface plasmon has a larger probability of being scattered out by the holes. This measurement yields the scattering cross section of the holes. Moreover the surface-plasmon contribution increases with hole density, as more holes are available to excite surface plasmons. The results for the most dense sample are not consistent with the other samples, which implies that the quasicylindrical wave contribution might be visible in correlation functions.

We believe the application of speckle correlation functions to plasmonics can be very valuable. The experiments give insight into both the surface-plasmon excitation and outcoupling by the holes. Moreover, it may be possible to study the influence of the quasicylindrical wave on the correlation functions in theory and experiments. Also, the combination of a constant background and a wavelength-dependent part is new compared to three-dimensional random media. ${ }^{7}$ Furthermore it is interesting that two different transmission processes can 
be separated using speckle correlation functions. It would be interesting to see if similar behavior is found when the threedimensional samples are made sufficiently thin compared to the transport mean free path or when these media are modified such that very short paths through the medium exist. ${ }^{23}$

\section{ACKNOWLEDGMENTS}

This work is part of the research program of the Foundation for Fundamental Research on Matter (FOM), which is part of the Netherlands Organisation for Scientific Research (NWO).
${ }^{1}$ T. W. Ebbesen, H. J. Lezec, H. F. Ghaemi, T. Thio, and P. A. Wolff, Nature (London) 391, 667 (1998).

${ }^{2}$ P. Lalanne, J. P. Hugonin, H. T. Liu, and B. Wang, Surf. Sci. Rep. 64, 453 (2009).

${ }^{3}$ F. J. García-Vidal, L. Martín-Moreno, T. W. Ebbesen, and L. Kuipers, Rev. Mod. Phys. 82, 729 (2010).

${ }^{4}$ S. Feng, C. Kane, P. A. Lee, and A. D. Stone, Phys. Rev. Lett. 61, 834 (1988).

${ }^{5}$ A. Z. Genack, Phys. Rev. Lett. 58, 2043 (1987).

${ }^{6}$ I. Freund, M. Rosenbluh, and S. Feng, Phys. Rev. Lett. 61, 2328 (1988).

${ }^{7}$ M. P. van Albada, J. F. de Boer, and A. Lagendijk, Phys. Rev. Lett. 64, 2787 (1990).

${ }^{8}$ S. Faez, P. M. Johnson, and A. Lagendijk, Phys. Rev. Lett. 103, 053903 (2009).

${ }^{9}$ N. Curry, P. Bondareff, M. Leclercq, N. F. van Hulst, R. Sapienza, S. Gigan, and S. Grésillon, Opt. Lett. 36, 3332 (2011).

${ }^{10}$ M. P. van Albada, B. A. van Tiggelen, A. Lagendijk, and A. Tip, Phys. Rev. Lett. 66, 3132 (1991).

${ }^{11}$ K. L. van der Molen, K. J. Klein Koerkamp, S. Enoch, F. B. Segerink, N. F. van Hulst, and L. Kuipers, Phys. Rev. B 72, 045421 (2005).
${ }^{12}$ J. A. Hutchison, D. M. O'Carroll, T. Schwartz, C. Genet, and T. W. Ebbesen, Angew. Chem. 123, 2133 (2011).

${ }^{13}$ F. van Beijnum, C. Rétif, C. B. Smiet, and M. P. van Exter, Opt. Lett. 36, 3666 (2011).

${ }^{14}$ L. Isserlis, Biometrika 12, 134 (1918).

${ }^{15}$ I. Edrei and M. Kaveh, Phys. Rev. B 38, 950 (1988).

${ }^{16}$ O. L. Muskens, T. van der Beek, and A. Lagendijk, Phys. Rev. B 84, 035106 (2011).

${ }^{17}$ H. T. Liu and P. Lalanne, Nature (London) 452, 728 (2008).

${ }^{18}$ W. Dai and C. M. Soukoulis, Phys. Rev. B 80, 155407 (2009).

${ }^{19}$ A. Y. Nikitin, F. J. García-Vidal, and L. Martín-Moreno, Phys. Rev. Lett. 105, 073902 (2010).

${ }^{20}$ L. Yin, V. K. Vlasko-Vlasov, J. Pearson, J. M. Hiller, J. Hua, U. Welp, D. E. Brown, and C. W. Kimball, Nano Lett. 5, 1399 (2005).

${ }^{21}$ P. B. Johnson and R. W. Christy, Phys. Rev. B 6, 4370 (1972).

${ }^{22}$ E. D. Palik, Handbook of Optical Constants of Solids (Academic, Orlando,1985).

${ }^{23}$ P. Barthelemy, J. Bertolotti, and D. S. Wiersma, Nature (London) 453, 495 (2008). 